\title{
THE RAYLEIGH POLYNOMIAL
}

\section{NAND KISHORE}

1. Introduction. Let $J_{v}(z)$ be the Bessel function of the first kind, and denote the zeros of $z^{-\nu} J_{\nu}(z)$ by $j_{\nu, m}, m=1,2, \cdots$, where $\left|\operatorname{Re}\left(j_{v, m}\right)\right| \leqq\left|\operatorname{Re}\left(j_{v, m+1}\right)\right|$. The Rayleigh function $\sigma_{2 n}(\nu)$ and the polynomial $\phi_{2 n}(\nu)$ of order $2 n$ are defined by

$$
\begin{aligned}
& \sigma_{2 n}(\nu)=\sum_{m=1}^{\infty}\left(j_{\nu, m}\right)^{-2 n}, \quad n=1,2,3, \cdots ; \\
& \phi_{2 n}(\nu)=4^{n} \prod_{k=1}^{n}(\nu+k)^{[n / k]} \sigma_{2 n}(\nu),
\end{aligned}
$$

where $[x]$ denotes the greatest integer $\leqq x$. Rayleigh functions and polynomials of odd orders are identically zero. The function $\sigma_{2 n}(\nu)$ has been the subject of a number of investigations [3, p. 502]. Properties of $\sigma_{2 n}(\nu)$ have been discussed in a previous paper [1].

The object of the present paper is to discuss properties of the polynomial $\phi_{2 n}(\nu)$. In this discussion we shall need three results from [1]. Formulas (3), (4) and (5) of the present paper are taken from [1], and repeated here for the convenience of the reader.

$$
\begin{aligned}
z J_{\nu+1}(z) J_{\nu}^{-1}(z) & =2 \sum_{n=1}^{\infty} \sigma_{2 n}(\nu) z^{2 n} \\
(\nu+n) \sigma_{2 n}(\nu) & =\sum_{k=1}^{n-1} \sigma_{2 k}(\nu) \sigma_{2 n-2 k}(\nu), \\
\text { (5) } \quad \sum_{k=1}^{n}(-1)^{k-1} 4^{k}(k !)^{2}\left(\begin{array}{c}
n \\
k
\end{array}\right)\left(\begin{array}{c}
\nu+n \\
k
\end{array}\right) \sigma_{2 k}(\nu) & =n .
\end{aligned}
$$

2. Polynomials. The Rayleigh polynomials may be constructed from (3), (4) or (5) by using (2). We list here the first eight polynomials:

$$
\begin{aligned}
\phi_{2}(\nu) & =1, \quad \phi_{4}(\nu)=1, \quad \phi_{6}(\nu)=2 \\
\phi_{8}(\nu) & =5 \nu+11, \quad \phi_{10}(\nu)=14 \nu+38 \\
\phi_{12}(\nu) & =42 \nu^{8}+362 \nu^{2}+1026 \nu+946 \\
\phi_{14}(\nu) & =132 \nu^{3}+1316 \nu^{2}+4324 \nu+4580 \\
\phi_{16}(\nu) & =429 \nu^{5}+7640 \nu^{4}+53752 \nu^{3}+185430 \nu^{2}+311387 \nu+202738
\end{aligned}
$$

Received by the editors January 30, 1963 and, in revised form, August 1, 1963. 
For the next four polynomials the reader is referred to [2].

3. The degree. Let $d_{n}$ be the degree of $\phi_{2 n}(\nu)$. Then if we substitute (2) in (4) and use induction on $n$ the following is obtained:

$$
d_{n}=1-2 n+\sum_{k=1}^{n}\left[\frac{n}{k}\right]
$$

(see [2]). Take $d_{n-1}$ and $d_{n}$, the degrees of two consecutive polynomials $\phi_{2 n-2}(\nu)$ and $\phi_{2 n}(\nu)$, then

$$
\begin{aligned}
d_{n}-d_{n-1} & =1-2 n+\sum_{k=1}^{\infty}\left[\frac{n}{k}\right]-\left\{1-2(n-1)+\sum_{k=1}^{\infty}\left[\frac{n-1}{k}\right]\right\} \\
& =-2+\sum_{k=1}^{\infty}\left\{\left[\frac{n}{k}\right]-\left[\frac{n-1}{k}\right]\right\} .
\end{aligned}
$$

However, the quantity on the right side equals the number of nontrivial divisors of $n$; therefore,

$$
d_{n}-d_{n-1}=\text { the number of nontrivial divisors of } n \text {. }
$$

From (7) we get the following:

$$
d_{p}=d_{p-1}, \quad \text { where } p \text { is a prime. }
$$

4. Coefficients. We introduce here a symbol $\epsilon(r, k, n)$ which is defined by the following relation

$$
\epsilon(r, k, n)=[n / r]-[k / r]-[(n-k) / r] .
$$

It is seen that the value of $\epsilon(r, k, n)$ is either 0 or 1 . In particular,

$$
\epsilon(1, k, n)=\epsilon(k, k, n)=0 .
$$

Now suppose we write $\phi_{2 n}(\nu)$ as

$$
\phi_{2 n}(\nu)=\sum_{k=0}^{d_{n}} a_{n, k} \nu^{k},
$$

then the main property of the coefficients $a_{n, k}$ of $\nu^{k}$ may be stated in the following

THEOREM I. Every coefficient $a_{n, k}$ of $\nu^{k}$ in the polynomial $\phi_{2 n}(\nu)$ is a positive integer.

Proof. Observe that (4) may be written as

$$
(\nu+n) \sigma_{2 n}(\nu)=\sum_{k=1}^{[n / 2]} \alpha_{k} \sigma_{2 k}(\nu) \sigma_{2 n-2 k}(\nu)
$$


where

$$
\alpha_{k}=2, k<[n / 2] ; \quad \alpha_{[n / 2]}= \begin{cases}2 & \text { if } n \text { is odd } \\ 1 & \text { otherwise. }\end{cases}
$$

Substitute (2) in (11). Then since

$$
\begin{aligned}
\prod_{r=1}^{k}(\nu+r)^{-[k / r]} & =\prod_{r=1}^{n-k}(\nu+r)^{-[k / r]}, & k \leqq[n / 2] ; \\
\prod_{r=n-k+1}^{n-1}(\nu+r)^{[n / r]} & =\prod_{r=n-k+1}^{n-1}(\nu+r), & k \leqq[n / 2],
\end{aligned}
$$

we obtain the following:

$$
\phi_{2 n}(\nu)=\sum_{k=1}^{[n / 2]} \alpha_{k} \prod_{r=2}^{[n / 2]}(\nu+r)^{\epsilon(r, k, n)} \prod_{r=n-k+1}^{n-1}(\nu+r) \phi_{2 k} \phi_{2 n-2 k}(\nu) .
$$

If we now consider (12) with induction on $n$ the required result is obtained.

5. Real zeros. In this section we shall prove that all real zeros of $\phi_{2 n}(\nu)$ lie in a certain interval. First we see that in consideration of (12) and induction on $n$ the following inequality is valid:

$$
\phi_{2 n}(\nu)>0, \text { if } \nu \geqq-2 .
$$

We note that (13) gives an upper bound -2 for the set of all real zeros of $\phi_{2 n}(\nu)$. Next we shall determine a lower bound for the same set, and then combine the results in a theorem. We begin with

$$
\phi_{2 n}(-s) \neq 0 \text {, if } s \text { is a positive integer and } s \mid n \text {. }
$$

To prove this, consider (3):

$$
\begin{aligned}
2 \sum_{k=1}^{\infty} \sigma_{2 k}(\nu) z^{2 k} & =z J_{\nu+1}(z) J_{\nu}^{-1}(z) \\
& =z\left\{\sum_{k=0}^{\infty} \frac{(-1)^{k}(z / 2)^{\nu+1+2 k}}{k ! \Gamma(\nu+2+k)}\right\}\left\{\sum_{k=0}^{\infty} \frac{(-1)^{k}(z / 2)^{\nu+2 k}}{k ! \Gamma(\nu+1+k)}\right\}^{-1} \\
& =\frac{1}{2}\left\{\sum_{k=0}^{\infty} \frac{(-1)^{k} z^{2 k+2}}{k !(\nu)_{k+1} 4^{k}}\right\}\left\{\sum_{k=0}^{\infty} \frac{(-1)^{k} z^{2 k}}{k !(\nu)_{k} 4^{k}}\right\}^{-1},
\end{aligned}
$$

where $(\nu)_{m}=(\nu+1)(\nu+2) \cdots(\nu+m)$, and $(\nu)_{0}=1$. Substitute $z=t^{1 / 2 s}(\nu+s)^{1 / 2 s}$ in the above, then 


$$
\begin{aligned}
& 4 \sum_{k=1}^{\infty} \sigma_{2 k}(\nu) t^{k / s}(\nu+s)^{k / s} \\
& \quad=\left\{\sum_{k=0}^{\infty} \frac{(-1)^{k} t^{(k+1) / s}(\nu+s)^{(k+1) / \varepsilon}}{k !(\nu)_{k+1} 4^{k}}\right\}\left\{\sum_{k=0}^{\infty} \frac{(-1)^{k} t^{k / s}(\nu+s)^{k / s}}{k !(\nu)_{k} 4^{k}}\right\}^{-1} .
\end{aligned}
$$

Now if we let $\nu \rightarrow-s$, then in view of (2) all terms of the left side, except those for which $k$ is a multiple of $s$, become zero. Similarly, all quantities on the right except one from the first series and two from the second vanish. Hence after a simplification we have

$$
\sum_{m=1}^{\infty} t^{m} \lim _{\nu \rightarrow-s}(\nu+s)^{m} \sigma_{2 m s}(\nu)=\frac{s t}{s !(s-1) ! 4^{s}}\left\{1-\frac{t}{s !(s-1) ! 4^{s}}\right\}^{-1} .
$$

Equating the coefficients of $t^{m}$ on two sides we get

$$
\lim _{\nu \rightarrow-8}(\nu+s)^{m} \sigma_{2 m s}(\nu)=s\left\{s !(s-1) ! 4^{s}\right\}^{-m} .
$$

Clearly (14) is true in view of (2) and (15). In fact, an explicit expression for $\phi_{2 m e}(-s)$ can be obtained. In particular,

$$
\begin{aligned}
\lim _{\nu \rightarrow-n}(\nu+n) \sigma_{2 n}(\nu) & =\{(n-1) !\}^{-2} 4^{-n} ; \\
\phi_{2 n}(-n) & =\{(n-1) !\}^{-2} \prod_{k=1}^{n-1}(k-n)^{[n / k]} .
\end{aligned}
$$

We shall now show that

$$
(\nu+n) \sigma_{2 n}(\nu)>0, \quad \nu \leqq-n .
$$

The proof of this inequality is by induction on $n$. We observe that $\sigma_{2}(\nu)<0$ if $\nu<-1$, and $\sigma_{4}(\nu)<0$ if $\nu<-2$. Suppose $\sigma_{2 k}(\nu)<0, \nu<-k$, for $k<n$. Then $\sigma_{2 k}(\nu)$ and $\sigma_{2 n-2 k}(\nu)$ are negative when $\nu<-k$ and $\nu<-(n-k)$, respectively; so, in particular, when $\nu<-n$. Therefore, for $k<n, \sigma_{2 k}(\nu) \sigma_{2 n-2 k}(\nu)>0$ when $\nu<-n$. Hence

$$
\sum_{k=1}^{n-1} \sigma_{2 k}(\nu) \sigma_{2 n-2 k}(\nu)>0, \quad \nu<-n .
$$

Using (4) we obtain

$$
\sigma_{2 n}(\nu)=(\nu+n)^{-1} \sum_{k=1}^{n-1} \sigma_{2 k}(\nu) \sigma_{2 n-2 k}(\nu)<0, \quad \nu<-n .
$$

This result and (16) then lead to (18).

LEMMA. If $\nu \leqq-n$, then $\phi_{2 n}(\nu)$ is positive or negative according as $[\sqrt{ } n]$ is odd or even. 
Proof. Substitute (2) in (18), then

$$
\phi_{2 n}(\nu) \prod_{k=1}^{n-1}(\nu+k)^{-[n / k]}>0, \quad \nu \leqq-n .
$$

This implies that for $\nu \leqq-n, \phi_{2 n}(\nu)$ and the product $\prod_{k=1}^{n-1}(\nu+k)^{-[n / k]}$ have like signs. However, for $\nu \leqq-n$, the product is positive or negative according as the sum $\sum_{k=1}^{n-1}[n / k]$ is even or odd; and this sum is even or odd according as $[\sqrt{ } n]$ is odd or even. This completes the proof.

The lemma implies that $\phi_{2 n}(\nu)$ does not have zeros $\leqq-n$. Consequently, we have the following

THEOREM II. All real roots of $\phi_{2 n}(\nu)=0$ lie in the interval $(-n,-2)$.

6. Congruences. We intend to prove two congruences for the Rayleigh polynomials. The first is

$$
\begin{aligned}
2 \sum_{k=1}^{s}(k-1) !\left(\begin{array}{c}
\nu+n-1 \\
k-1
\end{array}\right) \prod_{r=2}^{[n / 2]}(\nu+r)^{\epsilon(r, k, n)} \phi_{2 k}(\nu) \phi_{2 n-2 k}(\nu) \\
\equiv \phi_{2 n}(\nu)(\bmod (\nu+n-1)(\nu+n-2) \cdots(\nu+n-s))
\end{aligned}
$$

where $s \leqq[n / 2]-1$. To prove this congruence take (12) and observe that

$$
\prod_{r=n-k+1}^{n-1}(\nu+r)=(k-1) !\left(\begin{array}{c}
\nu+n-1 \\
k-1
\end{array}\right)
$$

then (12) may be written as

$$
\phi_{2 n}(\nu)=\prod_{k=1}^{[n / 2]} \alpha_{k} T_{k}
$$

where

$$
T_{k}=(k-1) !\left(\begin{array}{c}
\nu+n-1 \\
k-1
\end{array}\right) \prod_{r=2}^{[n / 2]}(\nu+r)^{\epsilon(r, k, n)} \phi_{2 k}(\nu) \phi_{2 n-2 k}(\nu) .
$$

Then since $\alpha_{k}=2$ if $k<[n / 2]$, (20) may be written as

$$
\phi_{2 n}(\nu)=2 \sum_{k=1}^{s} T_{k}+\sum_{k=s+1}^{[n / 2]} \alpha_{k} T_{k}, \quad s \leqq\left[\frac{n}{2}\right]-1 .
$$

However,

$$
\sum_{k=++1}^{[n / 2]} \alpha_{k} T_{k} \equiv 0(\bmod (\nu+n-1)(\nu+n-2) \cdots(\nu+n-s)) .
$$


Hence

$$
\phi_{2 n}(\nu) \equiv 2 \sum_{k=1}^{\dot{1}} T_{k}(\bmod (\nu+n-1)(\nu+n-2) \cdots(\nu+n-s))
$$

which is (19). Substitute $s=1$ in (19), and observe that for $r>1$, $\epsilon(r, 1, n)$ equals 1 or 0 according as $r \mid n$ or $r \nmid n$. Then

$$
\phi_{2 n}(\nu) \equiv 2 \prod_{\delta}(\nu+\delta) \phi_{2 n-2}(\nu)(\bmod (\nu+n-1))
$$

where $\delta$ ranges over the proper divisors of $n$. From (21) we get the following

$$
\phi_{2 n}(\nu) \equiv 2 \prod_{\delta}(\delta+1-n) \phi_{2 n-2}(\nu)(\bmod (\nu+n-1)) ;
$$

(23) $\phi_{2 n}(\nu) \equiv 2 \phi_{2 n-2}(\nu)(\bmod (\nu+n-1))$, if $n$ is an odd prime.

The second congruence for the polynomials $\phi_{2 n}(\nu)$ is

$$
\begin{aligned}
n^{-1} \sum_{k=1}^{\dot{1}}\left\{P_{2 k}(\nu)\right. & \left.+P_{2 n-2 s+2 k-2}(\nu)\right\} \\
\equiv & (-1)^{n}(n-1) ! \phi_{2 n}(\nu) \\
& +\prod_{r=1}^{[n / 2]}(\nu+r)^{[n / r]-1}(\bmod (\nu+n) \cdots(\nu+n-s))
\end{aligned}
$$

where

$$
P_{2 m}(\nu)=(-1)^{m-1}(m !)^{2}\left(\begin{array}{c}
n \\
m
\end{array}\right)\left(\begin{array}{c}
\nu+n \\
m
\end{array}\right) \phi_{2 m}(\nu) \prod_{r=1}^{[n / 2]}(\nu+r)^{[n / r]-[m / r]-1},
$$

and

$$
0 \leqq s<[n / 2] \text {. }
$$

This congruence is obtained from (5). For, substitution of (2) in (5) yields

$$
\sum_{k=1}^{n}(-1)^{k-1}(k !)^{2}\left(\begin{array}{c}
n \\
k
\end{array}\right)\left(\begin{array}{c}
\nu+n \\
k
\end{array}\right) \phi_{2 k}(\nu) \prod_{s=1}^{k}(\nu+s)^{-[k / s]=n .}
$$

Now multiply the above by

$$
\prod_{r=1}^{n}(\nu+r)^{[n / r]-1},
$$

and observe that $[n / r]-[k / r]-1 \geqq 0$, for $k \leqq n-1, r \leqq n$. Then 


$$
\begin{aligned}
& (-1)^{n}(n-1) ! \phi_{2 n}(\nu)+\prod_{r=1}^{n}(\nu+r)^{[n / r]-1} \\
& =n^{-1} \sum_{k=1}^{n-1}(-1)^{k-1}(k !)^{2}\left(\begin{array}{c}
n \\
k
\end{array}\right)\left(\begin{array}{c}
\nu+n \\
k
\end{array}\right) \phi_{2 k}(\nu) \prod_{r=1}^{n}(\nu+r)^{[n / r]-[k / r]-1} .
\end{aligned}
$$

The above relation leads to the required congruence. If we set $s=0$ in (24) then

$$
(-1)^{n-1}(n-1) ! \phi_{2 n}(\nu) \equiv \prod_{r=1}^{[n / 2]}(\nu+r)^{[n / r]-1}(\bmod (\nu+n))
$$

or

$$
\begin{aligned}
(\nu+1)(\nu+2) \cdots(\nu+n-1) & \phi_{2 n}(\nu) \\
& \equiv \prod_{r=1}^{[n / 2]}(\nu+r)^{[n / r]-1}(\bmod (\nu+n)) .
\end{aligned}
$$

\section{BIBLIOGRAPHY}

1. N. Kishore, The Rayleigh function, Proc. Amer. Math. Soc. 14 (1963), 527-533.

2. D. H. Lehmer, Zeros of the Bessel function $J_{\gamma}(x)$, MTAC 1 (1943-1945), 405407.

3. G. N. Watson, A treatise on the theory of Bessel functions, Cambridge Univ. Press, Cambridge, 1958.

UNIVERSITY OF TOLEDO 